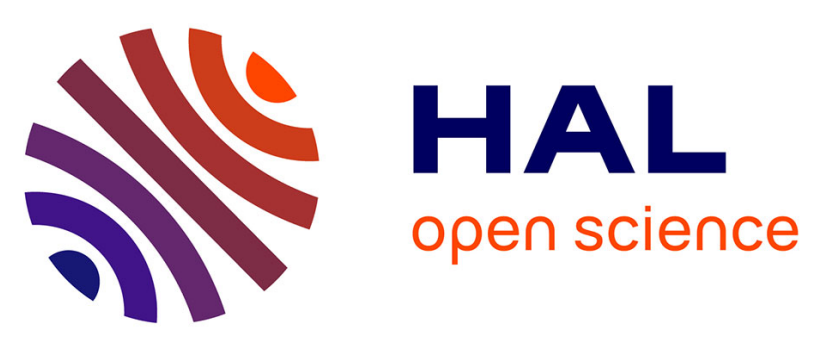

\title{
Stérilités mâle et femelle dans le genre Vitis. I. Modélisation de leur hérédité
}

Alain Carbonneau

\section{To cite this version:}

Alain Carbonneau. Stérilités mâle et femelle dans le genre Vitis. I. Modélisation de leur hérédité. Agronomie, 1983, 3 (7), pp.635-644. hal-00884553

\section{HAL Id: hal-00884553 https://hal.science/hal-00884553}

Submitted on 1 Jan 1983

HAL is a multi-disciplinary open access archive for the deposit and dissemination of scientific research documents, whether they are published or not. The documents may come from teaching and research institutions in France or abroad, or from public or private research centers.
L'archive ouverte pluridisciplinaire HAL, est destinée au dépôt et à la diffusion de documents scientifiques de niveau recherche, publiés ou non, émanant des établissements d'enseignement et de recherche français ou étrangers, des laboratoires publics ou privés. 


\section{Stérilités mâle et femelle dans le genre Vitis. I. Modéli- sation de leur hérédité}

Alain CARBONNEAU

I.N.R.A., Station de Recherches de Viticulture, Centre de Recherches de Bordeaux, F 33140 Pont-de-la-Maye

RÉSUMÉ

En fonction de données nouvelles concernant les caractères sexuels dans le genre Vitis, en particulier la preuve de l'apparition de mâles dans des descendances de croisements entre hermaphrodites, une modélisation générale de l'hérédité des stérilités mâle et femelle est développée.

Les résultats fournis par le meilleur modèle expliquent les observations effectuées jusqu'à ce jour et mettent en exergue des phénomènes de multiallélisme et d'épistasie.

Mots clés additionnels : Sexe, fleur, vigne, multiallélisme, épistasie.

Male and female sterility in the genus Vitis. I. Modeling of their inheritance.

A general model of the inheritance of male and female sterility in Vitis has been developed starting from new data on sexual characters in the genus Vitis, and particularly from evidence of the occurrence of males in progenies from crosses between hermaphrodites.

The results given by the best model explain the present observations and indicate multiallelic and epistatic phenomena.

Additional key words : Sex, flower, grapevine, multiallelism, epistasy.

\section{INTRODUCTION}

L'étude de l'hérédité des stérilités mâle et femelle chez la vigne conduit à des réflexions théoriques sur la structure du génome des plantes supérieures, ce qui peut éventuellement éclairer l'origine des variétés de vigne, orienter les choix de programmes de sélection pour certains caractères du moins, guider l'utilisation des stérilités mâle et femelle dans les croisements et permettre de mieux prévoir les fréquences d'apparition des types sexuels intéressants dans les descendances.

La vigne possède 3 types sexuels à l'intérieur desquels des variantes morphologiques existent, tous les types de fleurs présentant étamines et pistils (LEVADOUX, 1946) :

— individus à fleurs hermaphrodites ;

— individus à fleurs femelles donc à stérilité mâle ;

— individus à fleurs mâles donc à stérilité femelle.

La stérilité mâle se caractérise par des étamines courtes et par une dégénérescence du pollen au stade de l'isolement de la microspore ; la stérilité femelle par un pistil peu proéminent et par un avortement du sac embryonnaire à un stade peu déterminé après la méiose.

La stérilité mâle est très stable en fonction des conditions de milieu ou de l'état physiologique de la plante ; la stérilité femelle est stable en conditions normales mais peut être surmontée par des traitements répétés de la fleur au moyen de cytokinines (Benzyl amino purine), voire occasionnellement par une vigueur importante de la plante (NEGI \& Olmo, 1966, 1971 ; DoAzan \& Cuellar, 1970).

Cytologiquement et physiologiquement, ces 2 types de stérilités semblent donc faire appel à des mécanismes très différents.

\section{OBSERVATIONS DE BASE SUR L'HÉRÉDITÉ DES STERILITÉS MÂLE ET FEMELLE CHEZ LA VIGNE}

Depuis le début, au siècle dernier, des premiers programmes d'hybridation chez la vigne, un certain nombre d'observations portant sur quelques millions de descendants sont considérées comme sûres par l'ensemble des sélectionneurs :

- croisements mâles $\times$ hermaphrodites ou femelles : prédominance générale d'individus mâles dans l'ensemble des descendances par rapport aux types hermaphrodite ou femelle;

- croisements hermaphrodites $\times$ hermaphrodites ou femelles : prédominance générale d'individus hermaphrodites dans l'ensemble des descendances par rapport aux types femelles, les types mâles étant présents dans des cas très rares. 
L'hérédité de la stérilité femelle présente donc un fort caractère de dominance, celle de la stérilité mâle une tendance à la récessivité. Souvent ces rapports de dominance font apparaître, d'une part, des rapports de disjonction très simples du type : 1-0,1-1, 3-1 et, d'autre part, un minimum de 3 génotypes mâles, de 2 génotypes hermaphrodites et d'un génotype femelle.

En outre, il n'a jamais été observé d'individu réunissant les 2 stérilités mâle et femelle. Il faut donc admettre l'existence d'un mécanisme efficace d'exclusion mutuelle de ces stérilités (DOAZAN \& RIVES, 1967). Il convient de noter ici que les notions de stérilités mâle et femelle sont à dissocier de la notion d'absence de fleurs en rapport avec les phénomènes d'initiation florale et non avec ceux de la différenciation sexuelle.

Peu de croisements réciproques ont été réalisés à grande échelle chez la vigne; de ce fait, aucun élément de base concernant une éventuelle hérédité cytoplasmique ne peut guider a priori tout effort de modélisation de l'hérédité de ces stérilités.

Un certain nombre d'observations complémentaires de ces notations classiques méritent d'être signalées:

La $1^{\text {re }}$ concerne le lien phénotypique qui semble exister assez souvent entre la stérilité mâle et le retard de mise à fruit des jeunes semis (ANTCLIFF, 1980) et qui impose d'étudier absolument l'ensemble des descendants d'un même croisement sur plusieurs cycles végétatifs. Des biais systématiques décelables au moyen du test de $\chi^{2}$ d'homogénéité peuvent apparaitre si de telles précautions ne sont pas prises, par exemple chez TSEKHMISTRENKO \& FILINOVA, 1972. Toutefois, certaines descendances présentent des déficits très importants en femelles malgré une observation suffisamment longue de la descendance (TRUEL P., comm. pers., 1977).

La $2^{\mathrm{e}}$ est l'apparition de mâles dans des croisements entre hermaphrodites notamment (NEGRUL, 1930; BREIDER \& SCHEU, 1937 ; ANTCLIFF, 1980). Ceux-ci sont présents en proportion très faible inférieure à $1 \mathrm{p} .100$ ou $1 \mathrm{p} .1000$. De ce fait, l'hypothèse de contamination par du pollen mâle étranger, toujours possible, est à retenir en priorité (ANTCLIFF, 1980).

Toutefois, LOOMIS (1948), LoOMIS \& WILLIAMS (1954, 1957), dans le sous-genre Muscadinia, ont observé dans la descendance d'une autofécondation d'hermaphrodite l'apparition d'environ $1 / 4$ de mâles. Ce fait très curieux a été jusqu'à présent écarté des constatations propres au sousgenre Euvitis en prétextant que ces 2 sous-genres de Vitis possédaient des hérédités différentes, tout comme est différent le nombre de leurs chromosomes $(2 \mathrm{n}=38$ pour Euvitis ; $2 \mathrm{n}=40$ pour Muscadinia).

Le dernier fait nouveau concerne les résultats de TRUEL (comm. pers., 1977) et ceux non publiés de CARBONNEAU, Truel et DoAzan (1980 et 1981) qui figurent dans le tablcau 1 et qui sont complétés par les travaux ultérieurs de BRONNER (1981). Ceux-ci indiquent clairement l'apparition d'unc proportion élevée de mâles dans certaines descendances de croisements entre hermaphrodites ou entre hermaphrodites et femelles. L'ampleur de cette apparition, d'une

TABLEAU 1

Résultats de disjonctions relatives au cépage hermaphrodite "Counoise " induisant l'apparition de mâles dans des descendances de croisements avec d'autres hermaphrodites.

Segregation for sex in progeny from the hermaphrodite variety Counoise crossed with other hermaphrodites : occurrence of males.

\begin{tabular}{|c|c|c|c|c|c|c|c|c|}
\hline \multicolumn{4}{|c|}{ CROISEMENTS } & \multicolumn{3}{|c|}{ DISJONCTIONS } & \multicolumn{2}{|r|}{ OBSERVATIONS } \\
\hline Source & Type & & $\delta$ & $\phi$ & $q$ & $\begin{array}{l}\text { Hypothèse } \\
\text { classique }\end{array}$ & $x^{2}$ & \\
\hline TRUEL (1977) & $\begin{array}{l}\text { Counoise } \\
\text { droit }\end{array}$ & $\times \quad$ Plant & $0(0 \%)$ & $187(77 \%)$ & $55(23 \%)$ & $0-0,75-0,25$ & $0,12$ (NS à $5 \%)$ & $\begin{array}{l}\text { disjonction clas- } \\
\text { sique }\end{array}$ \\
\hline TRUEL (1977) & Counoise & $\times$ Exbrayat & $0(0 \%)$ & $34(72 \%)$ & $13(28 \%)$ & $0-0,75-0,25$ & $0,17$ (NS à $5 \%)$ & $\begin{array}{l}\text { disjonction clas- } \\
\text { sique }\end{array}$ \\
\hline TRUEL (1977) & $\begin{array}{l}\text { Counoise } \\
\text { Franc }\end{array}$ & $\times$ Cabernet & $30(22 \%)$ & $69(52 \%)$ & $35(26 \%)$ & $0,25-0,50-0,25$ & $0,48$ (NS à $5 \%)$ & $\begin{array}{l}25 \text { hermaphrodi- } \\
\text { tes sur } 69 \text { présen- } \\
\text { tent une très forte } \\
\text { coulure }\end{array}$ \\
\hline $\begin{array}{l}\text { CARBONNEAU et al. } \\
(1980)\left({ }^{*}\right)\end{array}$ & Counoise & $\times$ Aleatico & $9(60 \%)$ & $2(13 \%)$ & $4(27 \%)$ & - & - & $\begin{array}{l}\text { première série de } \\
\text { notations } \\
\text { (15 individus) }\end{array}$ \\
\hline $\begin{array}{l}\text { CARBONNEAU } e t \text { al. } \\
(1980)\left({ }^{*}\right)\end{array}$ & Counoise & $\times$ Aleatico & $12(57 \%)$ & $4(19 \%)$ & $5(24 \%)$ & - & - & $\begin{array}{l}\text { seconde série de } \\
\text { notations } \\
\text { ( } 21 \text { individus) }\end{array}$ \\
\hline $\begin{array}{l}\text { CARBONNEAU et al. } \\
\text { (1981) }\end{array}$ & Counoise & $\times$ Aleatico & $12(46 \%)$ & $8(31 \%)$ & $6(23 \%)$ & - & - & $\begin{array}{l}\text { troisième série de } \\
\text { notations } \\
\text { ( } 26 \text { individus). La } \\
\text { suite des observa- } \\
\text { tions est en cours. }\end{array}$ \\
\hline
\end{tabular}


part, et la répétition de cette observation, d'autre part, rendent indubitable que ce fait ne peut être dû à des erreurs de pollinisation ou d'appréciation. Les résultats de LOOMIS (1948) et de LOOMIS \& Williams (1954, 1957) sur Vitis rotundifolia concernent donc également Vitis vinifera variété «Counoise » par exemple. Il est donc indispensable de trouver un modèle génétique expliquant, d'une part, les disjonctions classiques et, d'autre part, l'apparition relativement exceptionnelle d'une combinaison allélique dominante dans une descendance de parents présentant une combinaison allélique récessive pour les mêmes fonctions.

\section{III. ÉTUDE CRITIQUE \\ DES MODĖLES GÉNÉTIQUES SIMPLES EXPLIQUANT LES DISJONCTIONS CLASSIQUES}

\section{A. Hypothèse de Valleau-Levadoux}

Dans le cadre du déterminisme génétique des caractères sexuels dans le genre Vitis, l'hypothèse qui actuellement est retenue et qui explique parfaitement les disjonctions classiques et l'absence d'asexués, est celle de VALLEAU (1916) reprise par LEVADOUX (1946) ; c'est en particulier l'opinion de DoAZAN \& Rives (1967) et de ANTClifF (1980).

Elle postule l'existence de 3 allèles d'un même gène : $M$, $\mathrm{H}, \mathrm{F}$, dans l'ordre décroissant de dominance. $M$ induit la formation d'individus mâles; $\mathbf{H}$ celle d'hermaphrodites ; $F$ celle de femelles. Du reste, il vaudrait mieux dire pour tenir compte des phénomènes de stérilités visibles sur les plans morphologiques et physiologiques : « $\mathbf{H}$, assure le développement des organes mâles et femelle de la fleur; $M$, n'assure que le rôle de $\mathrm{H}$ vis-à-vis des étamines ; F, n'assure que le rôle de $\mathrm{H}$ vis-à-vis du pistil $»$.

Toutefois, ce modèle n'explique pas l'apparition de mâles dans les croisements entre hermaphrodites notamment.

\section{B. Hypothèse d'OberLe}

Contrairement à VALLEAU (1916) et LEVADOUX (1946), OBERLE (1938) fonde son raisonnement sur des stérilités géniques, mâle. et femelle, situées dans des loci contigus. Son schéma est le suivant :

- $\mathrm{S}_{\mathrm{p}}$ (dominant) gouverne une étape du développement du pollen ; $s_{p}$ (récessif) inapte à assurer cette fonction ;

- $S_{o}$ (dominant) inhibe une étape du développement des ovules; $s_{\mathrm{o}}$ (récessif) inapte à assurer cette fonction.

Tout comme le modèle « $\mathrm{M}, \mathrm{H}, \mathrm{F}$ », ce schéma explique bien les disjonctions classiques. Toutefois, ce dernier ne peut exclure l'apparition d'individus asexués du type $\left(s_{p} s_{p} / S_{o} S_{o}\right)$ et $\left(s_{p} s_{p} / S_{0} s_{o}\right)$. Pour pallier cet inconvénient, OBERLE (1938) suppose un linkage très étroit entre le locus $\left(S_{p}, s_{p}\right)$ et le locus $\left(S_{o}, s_{o}\right)$.

Mais comme le font remarquer DOAZAN \& Rives (1967), ce linkage, si étroit soit-il, ne peut suffire pour expliquer l'absence universelle des génotypes asexués; il ne permet du reste pas de prévoir l'apparition de mâles dans les croisements entre hermaphrodites notamment.

\section{Etude synthétique des modèles géniques simples}

Afin d'éviter la simultanéité d'apparition des stérilités mâle et femelle, il est nécessaire de supposer que tous les allèles en cause sont situés dans un même locus du chromosome donc sans possibilité de recombinaison entre eux, ceci sans hypothèse sur la structure fine du locus.
Il serait possible de supposer que les génotypes induisant la stérilité totale sont létaux. Mais une telle hypothèse ne permet pas de résoudre les problèmes précités des déficits en femelles et d'apparition exceptionnelle des mâles.

Trois phénotypes devant être décrits, il est également nécessaire de supposer qu'il existe au moins 3 allèles responsables. Dans ce contexte, il est clair que seul le modèle de VALLEAU-LEVADOUX est capable d'expliquer les disjonctions classiques: $\mathrm{M}>\mathrm{H}>\mathrm{F}$.

Il est possible d'envisager que 4 allèles interviennent au même locus, non pas pour expliquer les disjonctions classiques puisqu'il y aurait redondance entre 2 allèles, mais pour préparer des hypothèses concernant notamment l'apparition de mâles dans les croisements entre hermaphrodites.

Quelles pourraient être les fonctions attribuables à ces allèles?

Tout d'abord, étant donné que les disjonctions «non classiques" font appel soit à des déficits de femelles par rapport aux hermaphrodites, soit à des déficits de femelles ou d'hermaphrodites compensés par l'apparition de mâles, il est logique de supposer que c'est la stérilité mâle qui présenterait une certaine variabilité allélique.

Le modèle le plus simple dans cette hypothèse et excluant les 2 stérilités à la fois serait :

$\mathrm{M}=$ allèle assurant fertilité mâle et stérilité femelle

$H=$ allèle assurant fertilité mâle et fertilité femelle

$\mathrm{F}_{\mathrm{m}}=1^{\mathrm{er}}$ allèle assurant stérilité mâle et fertilité femelle

$\mathrm{F}_{\mathrm{h}}=2^{\mathrm{e}}$ allèle assurant stérilité mâle et fertilité femelle.

Il s'agit d'établir la hiérarchie de dominance entre ces 4 allèles, étant entendu que $F_{m}$ et $F_{h}$ ne peuvent, en raison de leur fonction, présenter de hiérarchie de dominance entre eux.

Face à toutes ces conditions, il existe au maximum 6 possibilités.

Seule la loi suivante $\left(\mathrm{M}>\mathrm{H}>\mathrm{F}_{\mathrm{m}}, \mathrm{F}_{\mathrm{h}}\right)$ permet d'expliquer les disjonctions classiques. Elle est évidemment identique à l'hypothèse de VALLEAU-LEVADOUX si $F=\left(F_{m}, F_{h}\right)$ et est analogue à l'hypothèse d'OBERLE si on suppose par exemple que :

$$
\begin{array}{ll}
M=S_{p} S_{o}, & H=S_{p} \dot{s}, \\
F_{m}=s_{p} s_{o}, & F_{h}=s_{p} s_{o} .
\end{array}
$$

\section{Modèles simples faisant intervenir une hérédité cyto- plasmique}

Bien qu'aucune indication concrète ne laisse soupçonner l'intervention d'une hérédité cytoplasmique, il convient d'étudier ce fait sur le plan théorique en envisageant un nombre maximum de 2 mécanismes de stérilité cytoplasmique, l'une mâle, l'autre femelle.

Ces 2 hérédités cytoplasmiques, cytoplasmes mâle fertile et mâle stérile, d'une part, cytoplasmes femelle fertile et femelle stérile, d'autre part, ne peuvent en tout cas intervenir ensemble. En effet, il serait impossible d'obtenir des femelles à partir d'hermaphrodites autofécondés, puisqu'il n'y aurait pas le cytoplasme mâle stérile chez les descendants.

D'autre part, il est clair que l'intervention exclusive d'une seule hérédité cytoplasmique ne peut expliquér les disjonctions classiques. Il faut donc supposer une interaction «gène-cytoplasme », par exemple au minimum un couple de cytoplasmes portant sur le caractère mâle ou femelle et un couple allélique portant sur le caractère femelle ou mâle.

Enfin, afin d'éviter la présence d'individus asexués, il faut supposer que la stérilité génique portant sur un caractère est 
en fait un allèle de restauration de la stérilité cytoplasmique portant sur l'autre caractère, ou bien que c'est la stérilité cytoplasmique qui restaure la stérilité génique.

Compte tenu de toutes ces données, 8 cas peuvent se présenter :

1. Hérédité mâle cytoplasmique $(F, S)$, hérédité femelle génique $(X, x)$
1-1: stérilité femelle $=\mathrm{x}$, $x$ restaurant $S,(x, S) \rightarrow F$
$1-2$ : stérilité femelle $=x$ $S$ restaurant $x,(S, x) \rightarrow X$
1-3: stérilité femelle $=X$,
$X$ restaurant $S,(X, S) \rightarrow F$
1-3: stérilité femelle $=X$
$S$ restaurant $X,(S, X) \rightarrow X$

2. Hérédité mâle génique $\left(X^{\prime}, x^{\prime}\right)$, hérédité femelle cytoplasmique $\left(F, S^{\prime}\right)$

2-1: stérilité mâle $=x^{\prime}, \quad x^{\prime}$ restaurant $S^{\prime},\left(x^{\prime}, S^{\prime}\right) \rightarrow F^{\prime}$

2-2 : stérilité mâle $=x^{\prime}, \quad S^{\prime}$ restaurant $x^{\prime},\left(S^{\prime}, x^{\prime}\right) \rightarrow X^{\prime}$

2-3 : stérilité mâle $=X^{\prime}, \quad X^{\prime}$ restaurant $S^{\prime},\left(X^{\prime}, S^{\prime}\right) \rightarrow F^{\prime}$

2-4: stérilité mâle $=X^{\prime}, \quad S^{\prime}$ restaurant $X^{\prime},\left(S^{\prime}, X^{\prime}\right) \rightarrow x^{\prime}$

Aucune de ces combinaisons n'explique les disjonctions classiques, en particulier par déficits de génotypes mâles et hermaphrodites. Il faudrait donc faire intervenir d'autres gènes ou d'autres cytoplasmes ce qui compliquerait fortement la modélisation puisqu'alors plus de 4 allèles ou cytoplasmes seraient nécessaires. Il est donc plus logique de ne retenir qu'une influence chromosomique. Deux seuls modèles alléliques simples sont donc envisageables.

$$
\begin{gathered}
1-\mathrm{M}>\mathrm{H}>\mathrm{F} \\
2-\mathrm{M}>\mathrm{H}>\mathrm{F}_{\mathrm{m}}, \mathrm{F}_{\mathrm{h}} .
\end{gathered}
$$

\section{ETUDE CRITIQUE}

DE TOUS LES MODÈLES GÉNIQUES SIMPLES EXPLIQUANT L'ENSEMBLE DES DISJONCTIONS

Pour expliquer l'apparition de mâles dans les croisements entre hermaphrodites et entre hermaphrodite et femelle, ainsi que les forts déficits en femelles dans les croisements entre hermaphrodites ou entre hermaphrodite et femelle, il convient donc d'introduire au minimum un couple allélique supplémentaire qui puisse interagir et ségréger par rapport à l'une ou l'autre des séries alléliques précédemment établies, sans pour autant induire l'apparition d'asexués.

Soit $(E, e)$ ce couple allélique possédant donc une fonction épistatique sur les séries $(M, H, F)$ ou sur $(M, H$, $\left.F_{m}, F_{h}\right)$.

\section{A. Etude des interactions possibles entre $(\mathbf{E}$, e) et $(\mathbf{M}>\mathbf{H}>\mathbf{F})$}

L'effet épistatique de $(E, e)$ doit porter sur les allèles $H$ ou $\mathrm{F}$ et leur conférer la fonction de $\mathrm{M}$ ou $\mathrm{H}$, afin d'expliquer l'apparition des mâles ou hermaphrodites non prévisibles dans les disjonctions classiques. Cet effet épistatique ne peut logiquement porter sur $\mathbf{M}$ puisque les croisements faisant intervenir des mâles sont bien explicables par le modèle classique. D'autre part, cet effet épistatique ne peut être lié qu'à un effet de dosage de l'allèle $\mathrm{E}$ pour expliquer l'apparition de caractères dominants dans des croisements entre parents n'extériorisant pas ces derniers.

Compte tenu de ces observations 3 combinaisons sont possibles:

$$
1-(E+E), H=M \text { et }(E+E), F=H .
$$

Ce modèle est faux car, par exemple, certains croisements très fréquents entre hermaphrodites (FF/EE) et femelles (FF/ee) ne donnent que des femelles (FF/Ee), ceci en raison de l'effet du dosage de gènes portant sur $\mathrm{E}$.

$$
2-(E+E), F=M \text { et }(E+E), H=H
$$

(ce qui ne change rien ici).

Ce modèle'est également faux car, par exemple, certains croisements très fréquents entre mâles $(\mathrm{FF} / \mathrm{EE})$ et femelles (FF/ee) ne donnent que des femelles (FF/Ee).

$$
3-(E+E), H=M \text { et }(E+E), F=F
$$

(ce qui ne change rien ici).

Ce modèle explique les disjonctions classiques et l'apparition de mâles dans des croisements entre hermaphrodites ou entre hermaphrodites et femelles. Toutefois, il est incapable de prévoir de forts déficits en femelles tout à fait caractéristiques dans certains croisements entre hermaphrodites ou entre hermaphrodites et femelles, et ceci quel que soit le taux de recombinaison entre les 2 loci.

Il faut donc pour expliquer l'ensemble des disjonctions observées introduire d'autres allèles. Une $1^{\text {re }}$ idée consisterait à conserver le modèle $(\mathrm{M}>\mathrm{H}>\mathrm{F})$ avec la relation $(\mathrm{E}+\mathrm{E}), \mathrm{H}=\mathrm{M}$ et à lui adjoindre un autre couple allélique $\left(E^{\prime}, e^{\prime}\right)$ avec, par exemple, la relation supplémentaire $\left(E^{\prime}+E^{\prime}\right), F=H$. Mais ceci fait appel au total à 7 allèles et à 1 ou 2 effets épistatiques et de dosages concernant $E$ ou $E^{\prime}$. Une autre possibilité plus simple faisant intervenir 6 allèles est cnvisagéc ci-après.

B. Etude des interactions possibles entre $(E$, e) et $\left(\mathbf{M}>\mathbf{H}>\mathbf{F}_{\mathrm{m}}, \mathbf{F}_{\mathrm{h}}\right)$

Dans cettc hypothèse, l'effet épistatique de $(E$, e) doit porter sur $F_{m}$ et $F_{h}$ et non sur $H$ pour différencier ce modèle du précédent, l'allèle $M$ restant indépendant de $(E, e)$ pour les mêmes raisons qu'au préalable.

Dans un premier temps, il n'est pas indispensable de faire intervenir un effet de dosage pour $(\mathrm{E}, \mathrm{e})$.

Le modèle se résume donc ainsi :

$M>H>F_{m}, F_{h}$ avec $E, F_{m}=M$ et $E, F_{h}=H$.

Lc tableau 2 présente la matrice des 36 génotypes diploïdes possibles selon ce modèle : 23 mâles, 10 hermaphrodites, 3 femelles.

Le problème qui est posé à ce stade de l'étude est celui de la position du locus (E, e) par rapport au locus $\left(\mathrm{M}, \mathrm{H}, \mathrm{F}_{\mathrm{m}}, \mathrm{F}_{\mathrm{h}}\right)$. Cette analyse ne peut se faire que par simulation.

\section{Simulation de la distance génique entre $(E$, e) et $(M$,} $\mathbf{H}, \mathbf{F}_{\mathrm{m}}, \mathbf{F}_{\mathrm{h}}$ )

Six situations ont été envisagées afin de dégager les plus vraisemblables.

- $(\mathrm{E}$, e) est situé dans la même unité cistronique que $\left(\mathrm{M}, \mathrm{H}, \mathrm{F}_{\mathrm{m}}, \mathrm{F}_{\mathrm{h}}\right.$ ) avec un taux de recombinaison de 0 ; 


\section{TABLEAU 2}

Matrice de génotypes diplö̈des avec le phénotype correspondant selon le modèle: $(E, e / M),\left(E / F_{m}\right)>(E, e / H),\left(E / F_{h}\right)>\left(e / F_{m}\right),\left(e / F_{h}\right)$.

Matrix of diploid genotypes with the corresponding phenotypes according to the model: $(E, e / M),\left(E / F_{m}\right)>(E, e / H),\left(E / F_{h}\right)>\left(e / F_{m}\right),\left(e / F_{h}\right)$.

\begin{tabular}{|c|c|c|c|c|c|c|c|c|c|c|c|c|c|c|c|c|}
\hline & ME & & $\mathrm{Me}$ & & $\mathrm{HE}$ & & $\mathrm{He}$ & & $\mathrm{F}_{\mathrm{m}} \mathrm{E}$ & & $\mathrm{F}_{\mathrm{m}} \mathrm{e}$ & & $F_{h} E$ & & $\mathrm{~F}_{\mathrm{h}} \mathrm{e}$ & \\
\hline \multirow[t]{2}{*}{ ME } & $\frac{\mathrm{ME}}{\mathrm{ME}}$ & & $\frac{\mathrm{ME}}{\mathrm{Me}}$ & & $\frac{\mathrm{ME}}{\mathrm{HE}}$ & & $\frac{\mathrm{ME}}{\mathrm{He}}$ & & $\frac{\mathrm{ME}}{\mathrm{F}_{\mathrm{m}} \mathrm{E}}$ & & $\frac{\mathrm{ME}}{\mathrm{F}_{\mathrm{m}} \mathrm{e}}$ & & $\frac{\mathrm{ME}}{\mathrm{F}_{\mathrm{h}} \mathrm{E}}$ & & $\frac{M E}{F_{h} \mathrm{e}}$ & \\
\hline & & $\delta$ & & $\delta$ & & $\delta$ & & $\delta$ & & $\delta$ & & $\delta$ & & $\delta$ & & $\delta$ \\
\hline \multirow[t]{2}{*}{$\mathrm{Me}$} & & & $\frac{\mathrm{Me}}{\mathrm{Me}}$ & & $\frac{\mathrm{Me}}{\mathrm{HE}}$ & & $\frac{\mathrm{Me}}{\mathrm{Hc}}$ & & $\frac{\mathrm{Me}}{\mathrm{F}_{\mathrm{m}} \mathrm{E}}$ & & $\frac{\mathrm{Me}}{\mathrm{F}_{\mathrm{m}} \mathrm{e}}$ & & $\frac{\mathrm{Me}}{\mathrm{F}_{\mathrm{h}} \mathrm{E}}$ & & $\frac{M c}{F_{h} c}=$ & \\
\hline & & & & $\delta$ & & $\delta$ & & $\delta$ & & $\delta$ & & $\delta$ & & $\delta$ & & $\delta$ \\
\hline \multirow[t]{2}{*}{$\mathrm{HE}$} & & & & & $\frac{\mathrm{HE}}{\mathrm{HE}}$ & & $\frac{\mathrm{HE}}{\mathrm{He}}$ & & $\frac{\mathrm{HE}}{\mathrm{F}-\mathrm{E}}$ & & $\frac{\mathrm{HE}}{\mathrm{F}}$ & & $\frac{\mathrm{HE}}{\mathrm{F}_{\mathrm{E}}}$ & & $\frac{\mathrm{HE}}{\mathrm{F}_{\mathrm{c}}}$ & \\
\hline & & & & & & $q$ & & $\phi$ & & $\delta$ & & $\delta$ & & $\Phi$ & & $\phi$ \\
\hline \multirow[t]{2}{*}{$\mathrm{He}$} & & & & & & & $\frac{\mathrm{He}}{\hat{\mathrm{He}}}$ & & $\frac{\mathrm{He}}{\mathrm{Fe}}$ & & $\frac{\mathrm{He}}{\mathrm{Fe}}$ & & $\frac{\mathrm{He}}{\mathrm{FF}}$ & & $\frac{\mathrm{He}}{\mathrm{Fe}}$ & \\
\hline & & & & & & & & $\phi$ & & $\delta$ & & $\phi$ & & $\$$ & & $\$$ \\
\hline \multirow[t]{2}{*}{$\mathrm{F}_{\mathrm{m}} \mathrm{E}$} & & & & & & & & & $\frac{F_{m} E}{F_{m} E}$ & & $\frac{F_{m} E}{F_{m}} \mathrm{e}$ & & $\frac{F_{m} E}{F_{k} E}$ & & $\frac{F_{m} E}{F_{F}}$ & \\
\hline & & & & & & & & & & $\delta$ & & $\delta$ & & $\delta$ & & $0^{*}$ \\
\hline \multirow[t]{2}{*}{$F_{m} c$} & & & & & & & & & & & $\frac{F_{m} e}{E}$ & & $\frac{F_{m} e}{F}$ & & $\frac{F_{m} c}{F}$ & \\
\hline & & & & & & & & & & & & $q$ & & $\delta$ & & $q$ \\
\hline \multirow[t]{2}{*}{$\mathrm{F}_{\mathrm{h}} \mathrm{E}$} & & & & & & & & & & & & & $\frac{F_{h} E}{F_{n}}$ & & $\frac{F_{\mathrm{h}} \mathrm{E}}{\mathrm{F}_{0}}$ & \\
\hline & & & & & & & & & & & & & & $\phi$ & & $\hat{\phi}$ \\
\hline $\mathrm{F}_{\mathrm{h}} \mathrm{e}$ & & & & & & & & & & & & & & & $\frac{F_{h} e}{F_{h} e}$ & \\
\hline
\end{tabular}

- $(E$, e) est situé sur le même chromosome que $(\mathrm{M}, \mathrm{H}$, $F_{m}, F_{h}$ ) avec des taux de recombinaison de 0,$1 ; 0,2 ; 0,3$; 0,4 ;

-- $(E, e)$ est situé sur un chromosome différent de celui de $\left(\mathrm{M}, \mathrm{H}, \mathrm{F}_{\mathrm{m}}, \mathrm{F}_{\mathrm{h}}\right)$ done avec un taux de recombinaison de 0,5 .

Le résultat de ces simulations effectuées sur ordinateur est consigné dans les tableaux $3,4,5$ et 6 .

Afin de pouvoir déterminer la valeur approchée la plus probable de ce taux de recombinaison, la seule voie consiste à étudier les disjonctions présentant des caractéristiques assez uniques de façon à réduire le nombre de possibilités théoriques fournies par le modèle.

Le $1^{\text {er }}$ exemple retenu concerne l'étude des croisements femelle par mâle dont la descendance ne comprend aucun hermaphrodite. D'après le modèle (tabl. 3), les disjonctions attendues sont de 3 ordres:

a) $0,5(\delta)$ et $0,5(\$)$ quel que soit le taux de recombinaison (TR) ;

b) $0,5(\delta)$ et $0,5(q)$ si TR $=0 ; 0,55(\delta)$ et $0,45(q)$ si $\mathrm{TR}=0,1 ; 0,6\left(\delta^{\star}\right)$ et $0,4(q)$ si TR $=0,2 ; 0,65\left(\delta^{\star}\right)$ et 0,35 () si TR $=0,3 ; 0,7(\delta)$ et $0,3(\%)$ si TR $=0,4 ; 0,75\left({ }^{\star}\right)$ et $0,25(q)$ si $\mathrm{TR}=0,5$;

c) $1(\delta)$ et $0(q)$ si TR $=0 ; 0,95\left(\delta^{*}\right)$ et $0,05(q) \mathrm{si}$ $\mathrm{TR}=0,1 ; 0,9(\delta)$ et $0,1(q)$ si TR $=0,2 ; 0,85\left(\delta^{\star}\right)$ et 0,15 (₹) $\mathrm{si} \mathrm{TR}=0,3 ; 0,8(\delta)$ et $0,2(₹)$ si $\mathrm{TR}=0,4 ; 0,75\left(\delta^{\star}\right)$ et $0,25(q)$ si $\mathrm{TR}=0,5$.
Certains résultats de TRUEL (comm. pers., 1977) obtenus sur plusieurs centaines de descendants font apparaître 3 groupes de disjonctions significativement différents à 5 p. 100 de risque. Le $1^{\text {er }}$ donne des proportions très voisines de $0,5\left(\sigma^{3}\right)$ et $0,5(q)$ ce qui ne permet pas de présager du taux de recombinaison (croisement de type $a$ ). Le $2^{\mathrm{c}}$ donne : $0,7\left(\delta^{\circ}\right)$ et $0,3(q)$, la valeur $0,3(q)$ étant certainement un minimum compte tenu des observations d'ANTCLIFF (1980); le taux de recombinaison le plus probable est donc 0,4 correspondant au croisement de type $b$, la valeur 0,3 n'étant pas exclusible à 5 p. 100 de risque. Le $3^{\text {e }}$ donne: $0,9\left(\delta^{*}\right)$ et $0,1(q)$, ce qui fait intervenir le croisement de type $c$, et la valeur la plus probable du taux de recombinaison de 0,2 , cette valeur étant un minimum compte tenu des observations d'ANTCLIFF (1980); la valeur de 0,3 n'est pas éliminable à 5 p. 100 de risque.

Le $2^{\mathrm{e}}$ exemple choisi envisage l'étude des croisements femelle par hermaphrodite dont la descendance comprend uniquement des hermaphrodites et des femelles. D'après le modèle (tabl. 4) les disjonctions attendues sont de 4 ordres :

a) $0,5(\$)$ et $0,5(q)$ quel que soit le taux de recombinaison ;

b) $0,5(\$)$ et $0,5(q)$ si $\mathrm{TR}=0 ; 0,55(\$)$ et $0,45(q) \mathrm{si}$ $\mathrm{TR}=0,1 ; 0,6(\phi)$ et $0,4(q)$ si $\mathrm{TR}=0,2 ; 0,65$ (\$) et 
TABLEAU 3

Rapports de disjonctions pour les croisements "femelle $\times$ mâle 》.

Segregation ratios for "female $\times$ male" crosses.

Taux de recombinaisons entre $\left(E\right.$, e) et $\left(M, H, F_{m}, F_{h}\right)$

\begin{tabular}{|c|c|c|c|c|c|c|c|c|c|c|c|c|c|c|c|c|c|}
\hline & 0 & & & 0,1 & & & 0,2 & & & 0,3 & & & 0,4 & & & 0,5 & \\
\hline$\delta$ & $\$$ & $q$ & $\delta$ & $\phi$ & $q$ & 0 & $\$$ & $q$ & $\delta$ & $\phi$ & $q$ & a & $\phi$ & $q$ & t & $\phi$ & $q$ \\
\hline 1 & $0^{7}$ & 0 & 1 & $0^{7}$ & 0 & 1 & $0^{7}$ & 0 & 1 & $0^{7}$ & 0 & 1 & $0^{7}$ & 0 & 1 & $0^{7}$ & 0 \\
\hline 1 & 0 & 0 & 0,95 & 0,05 & 0 & 0,9 & 0,1 & 0 & 0,85 & 0,15 & 0 & 0,8 & 0,2 & 0 & 0,75 & 0,25 & 0 \\
\hline 1 & 0 & 0 & 0,95 & 0 & 0,05 & 0,9 & 0 & 0,1 & 0,85 & 0 & 0,15 & 0,8 & 0 & 0,2 & 0,75 & 0 & 0,25 \\
\hline 0,75 & 0,25 & 0 & 0,75 & 0,25 & 0 & 0,75 & 0,25 & 0 & 0,75 & 0,25 & 0 & 0,75 & 0,25 & 0 & 0,75 & 0,25 & 0 \\
\hline 0,75 & 0,25 & 0 & 0,725 & 0,275 & 0 & 0,7 & 0,3 & 0 & 0,675 & 0,325 & 0 & 0,65 & 0,35 & 0 & 0,625 & 0,375 & 0 \\
\hline 0,75 & 0,25 & 0 & 0,725 & 0,225 & 0,05 & 0,7 & 0,2 & 0,1 & 0,675 & 0,175 & 0,15 & 0,65 & 0,15 & 0,2 & 0,625 & 0,125 & 0,25 \\
\hline 0,5 & 0,5 & 0 & 0,5 & 0,5 & 0 & 0,5 & 0,5 & 0 & 0,5 & 0,5 & 0 & 0,5 & 0,5 & 0 & 0,5 & 0,5 & 0 \\
\hline 0,5 & 0,5 & 0 & 0,55 & 0,45 & 0 & 0,6 & 0,4 & 0 & 0,65 & 0,35 & 0 & 0,7 & 0,3 & 0 & 0,75 & 0,25 & 0 \\
\hline 0,5 & 0,5 & 0 & 0,525 & 0,475 & 0 & 0,55 & 0,45 & 0 & 0,575 & 0,425 & 0 & 0,6 & 0,4 & 0 & 0,625 & 0,375 & 0 \\
\hline 0,5 & 0,5 & 0 & 0,5 & 0,45 & 0,05 & 0,5 & 0,4 & 0,1 & 0,5 & 0,35 & 0,15 & 0,5 & 0,3 & 0,2 & 0,5 & 0,25 & 0,25 \\
\hline 0,5 & 0,5 & 0 & 0,475 & 0,475 & 0,05 & 0,45 & 0,45 & 0,1 & 0,425 & 0,425 & 0,15 & 0,4 & 0,4 & 0,2 & 0,375 & 0,375 & 0,25 \\
\hline 0,5 & 0,5 & 0 & 0,45 & 0,5 & 0,05 & 0,4 & 0,5 & 0,1 & 0,35 & 0,5 & 0,15 & 0,3 & 0,5 & 0,2 & 0,25 & 0,5 & 0,25 \\
\hline 0,5 & 0 & 0,5 & 0,5 & 0 & 0,5 & 0,5 & 0 & 0,5 & 0,5 & 0 & 0,5 & 0,5 & 0 & 0,5 & 0,5 & 0 & 0,5 \\
\hline 0,5 & 0 & 0,5 & 0,55 & 0 & 0,45 & 0,6 & 0 & 0,4 & 0,65 & 0 & 0,35 & 0,7 & 0 & 0,3 & 0,75 & 0 & 0,25 \\
\hline 0,5 & 0 & 0,5 & 0,525 & 0,025 & 0,45 & 0,55 & 0,05 & 0,4 & 0,575 & 0,075 & 0,35 & 0,6 & 0,1 & 0,3 & 0,625 & 0,125 & 0,25 \\
\hline 0,5 & 0 & 0,5 & 0,5 & 0,05 & 0,45 & 0,5 & 0,1 & 0,4 & 0,5 & 0,15 & 0,35 & 0,5 & 0,2 & 0,3 & 0,5 & 0,25 & 0,25 \\
\hline 0,5 & 0 & 0,5 & 0,475 & 0,025 & 0,5 & 0,45 & 0,05 & 0,5 & 0,425 & 0,075 & 0,5 & 0,4 & 0,1 & 0,5 & 0,375 & 0,125 & 0,5 \\
\hline 0,5 & 0 & 0,5 & 0,45 & 0,05 & 0,5 & 0,4 & 0,1 & 0,5 & 0,35 & 0,15 & 0,5 & 0,3 & 0,2 & 0,5 & 0,25 & 0,25 & 0,5 \\
\hline 0,25 & 0,25 & 0,5 & 0,275 & 0,275 & 0,45 & 0,3 & 0,3 & 0,4 & 0,325 & 0,325 & 0,35 & 0,35 & 0,35 & 0,3 & 0,375 & 0,375 & 0,25 \\
\hline 0,25 & 0,25 & 0,5 & 0,275 & 0,225 & 0,5 & 0,3 & 0,2 & 0,5 & 0,325 & 0,175 & 0,5 & 0,35 & 0,15 & 0,5 & 0,375 & 0,125 & 0,5 \\
\hline 0 & 0,5 & 0,5 & 0,05 & 0,5 & 0,45 & 0,1 & 0,5 & 0,4 & 0,15 & 0,5 & 0,35 & 0,2 & 0,5 & 0,3 & 0,25 & 0,5 & 0,25 \\
\hline 0 & 0,5 & 0,5 & 0,05 & 0,45 & 0,5 & 0,1 & 0,4 & 0,5 & 0,15 & 0,35 & 0,5 & 0,2 & 0,3 & 0,5 & 0,25 & 0,25 & 0,5 \\
\hline
\end{tabular}

TABLEAU 4

Rapporis de disjonctions pour les croisements "femelle $\times$ hermaphrodite ".

Segregation ratios for "female $\times$ hermaphrodite" crosses.

\begin{tabular}{|c|c|c|c|c|c|c|c|c|c|c|c|c|c|c|c|c|c|}
\hline \multicolumn{18}{|c|}{ Taux de recombinaison entre $(E, e)$ et $\left(M, H, F_{m}, F_{h}\right)$} \\
\hline & 0 & & & 0,1 & & & 0,2 & & & 0,3 & & & 0,4 & & & 0,5 & \\
\hline$\theta$ & 0 & $q$ & 0 & $\delta$ & q & $\delta$ & 6 & 우 & 0 & $\delta$ & $q$ & $\sigma$ & $\sigma$ & $q$ & $\delta$ & $\delta$ & q \\
\hline 1 & $0^{+}$ & 0 & 1 & $0^{\mp}$ & 0 & 1 & $0^{7}$ & 0 & 1 & $0^{7}$ & 0 & 1 & $0^{7}$ & 0 & 1 & $0^{7}$ & 0 \\
\hline 0,5 & 0,5 & 0 & 0,5 & 0.5 & 0 & 0,5 & 0,5 & 0 & 0,5 & 0,5 & 0 & 0,5 & 0,5 & 0 & 0,5 & 0,5 & 0 \\
\hline 0,5 & 0,5 & 0 & 0,5 & 0,45 & 0,05 & 0,5 & 0,4 & 0,1 & 0,5 & 0,35 & 0,15 & 0,5 & 0,3 & 0,2 & 0,5 & 0,25 & 0,25 \\
\hline 0,5 & 0 & 0,5 & 0,5 & 0 & 0,5 & 0,5 & 0 & 0,5 & 0,5 & 0 & 0,5 & 0,5 & 0 & 0,5 & 0,5 & 0 & 0,5 \\
\hline 0,5 & 0 & 0,5 & 0,5 & 0,05 & 0,45 & 0,5 & 0,1 & 0,4 & 0,5 & 0,15 & 0,35 & 0,5 & 0,2 & 0,3 & 0,5 & 0,25 & 0,25 \\
\hline 0,25 & 0,75 & 0 & 0,25 & 0,75 & 0 & 0,25 & 0,75 & 0 & 0,25 & 0,75 & 0 & 0,25 & 0,75 & 0 & 0,25 & 0,75 & 0 \\
\hline 0,25 & 0,75 & 0 & 0,25 & 0,70 & 0,05 & 0,25 & 0,65 & 0,1 & 0,25 & 0,6 & 0,15 & 0,25 & 0,55 & 0,2 & 0,25 & 0,5 & 0,25 \\
\hline 0,25 & 0,25 & 0.5 & 0,25 & 0,25 & 0,5 & 0,25 & 0,25 & 0,5 & 0,25 & 0,25 & 0,5 & 0,25 & 0,25 & 0,5 & 0,25 & 0,25 & 0,5 \\
\hline 0,25 & 0,25 & 0.5 & 0,25 & 0,30 & 0,45 & 0,25 & 0,35 & 0,4 & 0,25 & 0,4 & 0,35 & 0,25 & 0,45 & 0,3 & 0,25 & 0,5 & 0,25 \\
\hline 0 & 1 & 0 & 0 & 1 & 0 & 0 & 1 & 0 & 0 & 1 & 0 & 0 & 1 & 0 & 0 & 1 & 0 \\
\hline 0 & 1 & 0 & 0 & 0,975 & 0,025 & 0 & 0,95 & 0,05 & 0 & 0,925 & 0,075 & 0 & 0,9 & 0,1 & 0 & 0,875 & 0,125 \\
\hline 0 & 1 & 0 & 0 & 0,95 & 0,05 & 0 & 0,9 & 0,1 & 0 & 0,85 & 0,15 & 0 & 0,8 & 0,2 & 0 & 0,75 & 0,25 \\
\hline 0 & 0,5 & 0.5 & 0 & 0,5 & 0,5 & 0 & 0,5 & 0,5 & 0 & 0,5 & 0,5 & 0 & 0,5 & 0,5 & 0 & 0,5 & 0,5 \\
\hline 0 & 0,5 & 0.5 & 0 & 0,55 & 0,45 & 0 & 0,6 & 0,4 & 0 & 0,65 & 0,35 & 0 & 0,7 & 0,3 & 0 & 0,75 & 0,25 \\
\hline
\end{tabular}

$0,35(q)$ si $\mathrm{TR}=0,3 ; 0,7(\$)$ et $0,3(q)$ si $\mathrm{TR}=0,4$; 0,75 (ф) et $0,25(q)$ si $\mathrm{TR}=0,5$;

c) $1(\$)$ et $0(q)$ si $\mathrm{TR}=0 ; 0,95(\phi)$ et $0,05(q)$ si $\mathrm{TR}=0,1 ; 0,9\left(\phi^{\circ}\right)$ et $0,1(\%)$ si $\mathrm{TR}=0,2 ; 0,85\left(\phi^{\dagger}\right)$ et $0,15(q)$ si $\mathrm{TR}=0,3 ; 0,8(\delta)$ et $0,2(\%)$ si $\mathrm{TR}=0,4$; $0,75(\$)$ et $0,25(\%)$ si TR $=0,5$;

d) 1 (ф) et 0 (q) si TR $=0 ; 0,975$ (ф) et 0,025 (q) si $\mathrm{TR}=0,1 ; 0,95(\$)$ et $0,05(q)$ si $\mathrm{TR}=0,2 ; 0,925(\delta)$ et $0,075(q)$ si $\mathrm{TR}=0,3 ; 0,9(\phi)$ et $0,1(q)$ si $\mathrm{TR}=0,4$; $0,875(\Varangle)$ et $0,125(\%)$ si $\mathrm{TR}=0,5$.
Le croisement du type $a$ est souvent rencontré et ne permet pas de déterminer la valeur du taux de recombinaison. En analysant certains résultats de TRUEL (comm. pers., 1977) et de GoRODEA \& NEAGU $(1977,1978)$ relatifs aux croisements avec la variété femelle «Bicane», il apparaît que seules des disjonctions correspondant au croisement de type $b$ sont explicables par le modèle. D'autres résultats de TRUEL (comm. pers., 1977) font appel à des croisements de type $c$ et $d$, mais ne comportent pas un nombre suffisant de descendants (moins de 50 individus par classe). Il convient 
donc de ne retenir que les croisements de type $b$. Dans ce contexte, TRUEL (comm. pers., 1977) observe les rapports suivants : $0,65(\$)$ et $0,35(\%)$, ce qui correspond à un taux de recombinaison de 0,3 , celui-ci étant un maximum. Gorodea \& NeAGU $(1977,1978)$ notent les proportions moyennes de $0,7(\emptyset)$ et $0,3(q)$, ce qui correspond à un taux de recombinaison de 0,4 , ce dernier étant un maximum.

Le $3^{\text {e }}$ exemple assez simple est illustré par le résultat de certaines autofécondations d'hermaphrodites. D'après le modèle (tabl. 5), les 4 séries de disjonctions suivantes sont attendues dans le cas où hermaphrodites et femelles sont observés dans les descendances.

a) 0,75 ( $\$$ ) et $0,25(q)$ quel que soit le taux de recombinaison:

b) $0,75(\phi)$ et $0,25(q)$ si $\mathrm{TR}=0 ; 0,7975(\phi)$ et $0,2025(\%) \quad$ si $\mathrm{TR}=0,1 ; 0,84\left(\delta^{\circ}\right) \quad$ et $0,16(\%)$ si $\mathrm{TR}=0,2 ; 0.8775(\phi)$ et $0,1225(\%)$ si $\mathrm{TR}=0,3$;

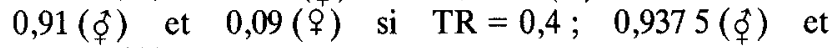
$0,0625(q)$ si $\mathrm{TR}=0,5$;

c) $1(\phi)$ et $0(q)$ si $\mathrm{TR}=0 ; 0,975(\phi)$ et $0,025(q) \mathrm{si}$ $\mathrm{TR}=0,1 ; 0,95(\phi)$ et $0,05(q)$ si $\mathrm{TR}=0,2 ; 0,925$ (ф) et $0,075(q)$ si $\mathrm{TR}=0,3 ; 0,9(\$)$ et $0,1(q)$ si $\mathrm{TR}=0,4$; $0,875(\$)$ et $0,125(q)$ si $\mathrm{TR}=0,5$;

d) $1(\phi)$ et $0(q)$ si TR $=0 ; 0,9975(\$)$ et $0,0025(q)$ si $\mathrm{TR}=0,1 ; 0,99(\phi)$ et $0,01(\%)$ si $\mathrm{TR}=0,2$; $0,9775(\phi)$ et $0,0225(q)$ si $\mathrm{TR}=0,3 ; 0,96(\phi)$ et $0,04(\%)$ si $\mathrm{TR}=0,4 ; 0,9375(\$)$ et $0,0625(\%)$ si $\mathrm{TR}=0,5$.

Le type $a$ est largement noté chez les sélectionneurs, mais ne permet pas de trouver le taux de recombinaison. BREIDER \& SCHEU (1937) observent une disjonction $0,88(\$)$ et $0,12(\$)$ dans l'autofécondation de la variété «Madeleine Céline», ce qui correspond bien au type $b$ pour TR $=0,3$, cette valeur étant ici un maximum. TRUEL (comm. pers., 1977) observe dans l'autofécondation de la variété « Jaoumet » le rapport $0,93(\hat{\phi})$ et $0,07(q)$, ce qui peut indiquer un croisement soit de type $b$ pour $\mathrm{TR}=0,5$ (ici maximum), soit de type $d$ pour TR $=0,5$ (ici maximum non dépassable), soit plus probablement de type $c$ pour $\mathrm{TR}=0,3$ (ici minimum).
Souvent également selon TRUEL (comm. pers., 1977), dans les croisements entre hermaphrodites à effectif suffisant et où apparaissent de très forts déficits en femelles par rapport au modèle classique (croisements faisant intervenir la variété «Aleatico »), un taux de recombinaison voisin de 0,3 expliquerait la majorité des résultats à un risque de $5 \mathrm{p}$. 100 .

Si l'on donne un bilan de ces diverses observations, il apparaît que le couple allélique (E,e) est situé sur le même chromosome que le locus $\left(\mathrm{M}, \mathrm{H}, \mathrm{F}_{\mathrm{m}}, \mathrm{F}_{\mathrm{h}}\right)$ avec un taux de recombinaison de 0,3 , ce qui constitue la valeur simple la plus probable. Eu égard au risque statistique déjà évoqué, les valeurs inférieures et supérieures se situeraient aux alentours de 0,2 et 0,4 respectivement.

\section{Exemples de détermination du génotype de quelques variétés « tests »}

Le modèle ainsi défini, il est vérifiable que les disjonctions classiques, sont parfaitement interprétables ainsi que l'apparition de mâles ou certains déficits en femelles dans les croisements entre hermaphrodites. Il est à noter également la présence de disjonctions non encore observées jusqu'à présent (tabl. 6). Quelques cas particuliers méritent toutefois d'être détaillés et permettent de déterminer d'ailleurs la formule génique de quelques variétés.

\section{Exemple de la variété femelle "Bicane"}

D’après le modèle proposé, il existe 3 génotypes femelles :

$$
\left(\mathrm{F}_{\mathrm{m}} \mathrm{e} / \mathrm{F}_{\mathrm{m}} \mathrm{e}\right),\left(\mathrm{F}_{\mathrm{m}} \mathrm{e} / \mathrm{F}_{\mathrm{h}} \mathrm{e}\right),\left(\mathrm{F}_{\mathrm{h}} \mathrm{e} / \mathrm{F}_{\mathrm{h}} \mathrm{e}\right) .
$$

Dans les croisements effectués avec la variété «Bicane " (voir chapitre précédent), faisant intervenir essentiellement des hermaphrodites comme parent mâle, il n'a pas été observé d'individu mâle dans les descendances, mais des disjonctions classiques faisant apparaitre uniquement des hermaphrodites, ou des hermaphrodites et des femelles à part égale, ou enfin une dominance d'hermaphrodites par rapport aux femelles (ce qui est le cas le plus général).

\section{TABLEAU 5}

Rapports de disjonctions pour les croisements " hermaphrodite $\times$ hermaphrodite " dont les autofécondations. Segregation ratios for " hermaphrodite $\times$ hermaphrodite" crosses including selfings.

Taux de recombinaison entre $(E, e)$ et $\left(M, H, F_{m}, F_{h}\right)$

\begin{tabular}{|c|c|c|c|c|c|c|c|c|c|c|c|c|c|c|c|c|c|c|c|}
\hline & & \multicolumn{3}{|c|}{0} & \multicolumn{3}{|c|}{0,1} & \multicolumn{3}{|c|}{0,2} & \multicolumn{3}{|c|}{0,3} & \multicolumn{3}{|c|}{0,4} & \multicolumn{3}{|c|}{0,5} \\
\hline & & $\delta$ & 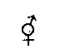 & q & $\delta$ & $\stackrel{\alpha}{\phi}$ & $q$ & $\delta$ & के & 우 & $\delta$ & ఫ & 우 & $\delta$ & $\not{t}$ & ㅇ & $\sigma$ & $\phi$ & 우 \\
\hline \multirow{13}{*}{ 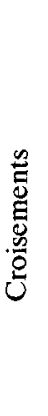 } & \multirow{13}{*}{ 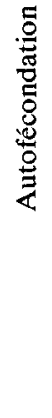 } & 0 & 1 & 0 & 0 & 1 & 0 & 0 & 1 & 0 & 0 & 1 & 0 & 0 & 1 & 0 & 0 & 1 & 0 \\
\hline & & 0 & 1 & 0 & 0 & 0,9975 & 0,0025 & 0 & 0,99 & 0,01 & 0 & 0,9775 & 0,0225 & 0 & 0,96 & 0,04 & 0 & 0,9375 & 0,0625 \\
\hline & & 0 & 1 & 0 & 0 & 0,975 & 0,025 & 0 & 0,95 & 0,05 & 0 & 0,925 & 0,075 & 0 & 0,9 & 0,1 & 0 & 0,875 & 0,125 \\
\hline & & 0 & 0,75 & 0,25 & 0 & 0,75 & 0,25 & 0 & 0,75 & 0,25 & 0 & 0,75 & 0,25 & 0 & 0,75 & 0,25 & 0 & 0,75 & 0,25 \\
\hline & & 0 & 0,75 & 0,25 & 0 & 0,7975 & 0,2025 & 0 & 0,84 & 0,16 & 0 & 0,8775 & 0,1225 & 0 & 0,91 & 0,09 & 0 & 0,9375 & 0,0625 \\
\hline & & 1 & 0 & 0 & 1 & 0 & 0 & 1 & 0 & 0 & 1 & 0 & 0 & 1 & 0 & 0 & 1 & 0 & 0 \\
\hline & & 0,5 & 0,5 & 0 & 0,5 & 0,5 & 0 & 0,5 & 0,5 & 0 & 0,5 & 0,5 & 0 & 0,5 & 0,5 & 0 & 0,5 & 0,5 & 0 \\
\hline & & 0,25 & 0,75 & 0 & 0,25 & 0,75 & 0 & 0,25 & 0,75 & 0 & 0,25 & 0,75 & 0 & 0,25 & 0,75 & 0 & 0,25 & 0,75 & 0 \\
\hline & & 0,25 & 0,75 & 0 & 0,25 & 0,725 & 0,025 & 0,25 & 0,7 & 0,05 & 0,25 & 0,675 & 0,075 & 0,25 & 0,65 & 0,1 & 0,25 & 0,625 & 0,125 \\
\hline & & 0,25 & 0,50 & 0,25 & 0,25 & 0,525 & 0,225 & 0,25 & 0,55 & 0,2 & 0,25 & 0,575 & 0,175 & 0,25 & 0,6 & 0,15 & 0,25 & 0,625 & 0,125 \\
\hline & & 0,25 & 0,50 & 0,25 & 0,25 & 0,50 & 0,25 & 0,25 & 0,50 & 0,25 & 0,25 & 0,50 & 0,25 & 0,25 & 0,50 & 0,25 & 0,25 & 0,50 & 0,25 \\
\hline & & 0 & 1 & 0 & 0 & 0,9775 & 0,0225 & 0 & 0,96 & 0,04 & 0 & 0,9475 & 0,0525 & 0 & 0,94 & 0,06 & 0 & 0,9375 & 0,0625 \\
\hline & & 0 & 0,75 & 0,25 & 0 & 0,775 & 0,225 & 0 & 0,8 & 0,2 & 0 & 0,825 & 0,175 & 0 & 0,85 & 0,15 & 0 & 0,875 & 0,125 \\
\hline
\end{tabular}


TABLEAU 6

Rapports de disjonctions pour les croisements " hermaphrodite $\times$ mâle ".

Segregation ratios for " hermaphrodite $\times$ male" crosses.

Taux de recombinaison entre $(E, e)$ et $\left(M, H, F_{m}, F_{h}\right)$

\begin{tabular}{|c|c|c|c|c|c|c|c|c|c|c|c|c|c|c|c|c|c|}
\hline & 0 & & & 0,1 & & & 0,2 & & & 0,3 & & & 0,4 & & & 0,5 & \\
\hline$\delta$ & $\phi$ & $q$ & 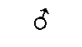 & 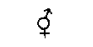 & q & $\delta$ & ᄒ & q & $\sigma$ & 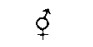 & $q$ & $\delta$ & $\phi$ & $q$ & $\delta$ & ț & q \\
\hline 1 & 0 & 0 & 1 & 0 & 0 & 1 & 0 & 0 & 1 & 0 & 0 & 1 & 0 & 0 & 1 & 0 & 0 \\
\hline 1 & 0 & 0 & 0,975 & 0,025 & 0 & 0,95 & 0,05 & 0 & 0,925 & 0,075 & 0 & 0,9 & 0,1 & 0 & 0,875 & 0,125 & 0 \\
\hline 1 & 0 & 0 & 0,975 & 0,0025 & 0,0225 & 0,95 & 0,01 & 0,04 & 0,925 & 0,0225 & 0,0525 & 0,9 & 0,04 & 0,06 & 0,875 & 0,0625 & 0,0625 \\
\hline 1 & 0 & 0 & 0,975 & 0 & 0,025 & 0,95 & 0 & 0,05 & 0,925 & 0 & 0,075 & 0,9 & 0 & 0,1 & 0,875 & 0 & 0,125 \\
\hline 1 & 0 & 0 & 0,95 & 0,05 & 0 & 0,9 & 0,1 & 0 & 0,85 & 0,15 & 0 & 0,8 & 0,2 & 0 & 0,75 & 0,25 & 0 \\
\hline 1 & 0 & 0 & 0,95 & 0,025 & 0,025 & 0,9 & 0,05 & 0,05 & 0,85 & 0,075 & 0,075 & 0,8 & 0,1 & 0,1 & 0,75 & 0,125 & 0,125 \\
\hline 0,75 & 0,25 & 0 & 0,75 & 0,25 & 0 & 0,75 & 0,25 & 0 & 0,75 & 0,25 & 0 & 0,75 & 0,25 & 0 & 0,75 & 0,25 & 0 \\
\hline 0,75 & 0,25 & 0 & 0,775 & 0,225 & 0 & 0,8 & 0,2 & 0 & 0,825 & 0,175 & 0 & 0,85 & 0,15 & 0 & 0,875 & 0,125 & 0 \\
\hline 0,75 & 0,25 & 0 & 0,725 & 0,275 & 0 & 0.7 & 0,3 & 0 & 0,675 & 0,325 & 0 & 0,65 & 0,35 & 0 & 0,625 & 0,375 & 0 \\
\hline 0,75 & 0,25 & 0 & 0,775 & 0,2025 & 0,0225 & 0,8 & 0,16 & 0,04 & 0,825 & 0,1225 & 0,0525 & 0,85 & 0,09 & 0,06 & 0,875 & 0,0625 & 0,0625 \\
\hline 0,75 & 0,25 & 0 & 0,75 & 0,225 & 0,025 & 0,75 & 0,2 & 0,05 & 0,75 & 0,175 & 0,075 & 0,75 & 0,15 & 0,1 & 0,75 & 0,125 & 0,125 \\
\hline 0,75 & 0,25 & 0 & 0,725 & 0,25 & 0,025 & 0,7 & 0,25 & 0,05 & 0,675 & 0,25 & 0,075 & 0,65 & 0,25 & 0,1 & 0,625 & 0,25 & 0,125 \\
\hline 0,75 & 0 & 0,25 & 0,75 & 0 & 0,25 & 0,75 & 0 & 0,25 & 0,75 & 0 & 0,25 & 0,75 & 0 & 0,25 & 0,75 & 0 & 0,25 \\
\hline 0,75 & 0 & 0,25 & 0,775 & 0,0225 & 0,2025 & 0,8 & 0,04 & 0,16 & 0,825 & 0,0525 & 0,1225 & 0,85 & 0,06 & 0,09 & 0,875 & 0,0625 & 0,0625 \\
\hline 0,75 & 0 & 0,25 & 0,775 & 0 & 0,225 & 0,8 & 0 & 0,2 & 0,825 & 0 & 0,175 & 0,85 & 0 & 0,15 & 0,875 & 0 & 0,125 \\
\hline 0,75 & 0 & 0,25 & 0,75 & 0,025 & 0,225 & 0,75 & 0,05 & 0,2 & 0,75 & 0,075 & 0,175 & 0,75 & 0,1 & 0,15 & 0,75 & 0,125 & 0,125 \\
\hline 0,5 & 0,5 & 0 & 0,5 & 0,5 & 0 & 0,5 & 0,5 & 0 & 0,5 & 0,5 & 0 & 0,5 & 0,5 & 0 & 0,5 & 0,5 & 0 \\
\hline 0,5 & 0,5 & 0 & 0,55 & 0,45 & 0 & 0,6 & 0,4 & 0 & 0,65 & 0,35 & 0 & 0,7 & 0,3 & 0 & 0,75 & 0,25 & 0 \\
\hline 0,5 & 0,5 & 0 & 0,525 & 0,475 & 0 & 0,55 & 0,45 & 0 & 0,575 & 0,425 & 0 & 0,6 & 0,4 & 0 & 0,625 & 0,375 & 0 \\
\hline 0,5 & 0,5 & 0 & 0,5 & 0,4975 & 0,0025 & 0,5 & 0,49 & 0,01 & 0,5 & 0,4775 & 0,0225 & 0,5 & 0,46 & 0,04 & 0,5 & 0,4375 & 0,0625 \\
\hline 0,5 & 0,5 & 0 & 0,5 & 0,4775 & 0,0225 & 0,5 & 0,46 & 0,04 & 0,5 & 0,4475 & 0,0525 & 0,5 & 0,44 & 0,06 & 0,5 & 0,4375 & 0,0625 \\
\hline 0,5 & 0,5 & 0 & 0,5 & 0,4750 & 0,025 & 0,5 & 0,45 & 0,05 & 0,5 & 0,425 & 0,075 & 0,5 & 0,4 & 0,1 & 0,5 & 0,375 & 0,125 \\
\hline 0,5 & 0,5 & 0 & 0,475 & 0,525 & 0 & 0,45 & 0,55 & 0 & 0,425 & 0,575 & 0 & 0,4 & 0,6 & 0 & 0,375 & 0,625 & 0 \\
\hline 0,5 & 0,5 & 0 & 0,475 & 0,5025 & 0,0225 & 0,45 & 0,51 & 0,04 & 0,425 & 0,5225 & 0,0525 & 0,4 & 0,54 & 0,06 & 0,375 & 0,5625 & 0,0625 \\
\hline 0,5 & 0,5 & 0 & 0,475 & 0,5 & 0,025 & 0,45 & 0,5 & 0,05 & 0,425 & 0,5 & 0,075 & 0,4 & 0,5 & 0,1 & 0,375 & 0,5 & 0,125 \\
\hline 0,5 & 0,5 & 0 & 0,45 & 0,55 & 0 & 0,4 & 0,6 & 0 & 0,35 & 0,65 & 0 & 0,3 & 0,7 & 0 & 0,25 & 0,75 & 0 \\
\hline 0,5 & 0,5 & 0 & 0,45 & 0,525 & 0,025 & 0,4 & 0,55 & 0,05 & 0,35 & 0,575 & 0,075 & 0,3 & 0,6 & 0,1 & 0,25 & 0,625 & 0,125 \\
\hline 0,5 & 0,25 & 0,25 & 0,5 & 0,25 & 0,25 & 0,5 & 0,25 & 0,25 & 0,5 & 0,25 & 0,25 & 0,5 & 0,25 & 0,25 & 0,5 & 0,25 & 0,25 \\
\hline 0,5 & 0,25 & 0,25 & 0,55 & 0,225 & 0,225 & 0,6 & 0,2 & 0,2 & 0,65 & 0,175 & 0,175 & 0,7 & 0,15 & 0,15 & 0,75 & 0,125 & 0,125 \\
\hline 0,5 & 0,25 & 0,25 & 0,525 & 0,25 & 0,225 & 0,55 & 0,25 & 0,2 & 0,575 & 0,25 & 0,175 & 0,6 & 0,25 & 0,15 & 0,625 & 0,25 & 0,125 \\
\hline 0,5 & 0,25 & 0,25 & 0,5 & 0,2975 & 0,2025 & 0,5 & 0,34 & 0,16 & 0,5 & 0,3775 & 0,1225 & 0,5 & 0,41 & 0,09 & 0,5 & 0,4375 & 0,0625 \\
\hline 0,5 & 0,25 & 0,25 & 0,5 & 0,275 & 0,225 & 0,5 & 0,3 & 0,2 & 0,5 & 0,325 & 0,175 & 0,5 & 0,35 & 0,15 & 0,5 & 0,375 & 0,125 \\
\hline 0,5 & 0,25 & 0,25 & 0,475 & 0,3 & 0,225 & 0,45 & 0,35 & 0,2 & 0,425 & 0,4 & 0,175 & 0,4 & 0,45 & 0,15 & 0,375 & 0,5 & 0,125 \\
\hline 0,5 & 0,25 & 0,25 & 0,475 & 0,275 & 0,25 & 0,45 & 0,3 & 0,25 & 0,425 & 0,325 & 0,25 & 0,4 & 0,35 & 0,25 & 0,375 & 0,375 & 0,25 \\
\hline 0,5 & 0,25 & 0,25 & 0,45 & 0,3 & 0,25 & 0,4 & 0,35 & 0,25 & 0,35 & 0,4 & 0,25 & 0,3 & 0,45 & 0,25 & 0,25 & 0,5 & 0,25 \\
\hline 0,25 & 0,75 & 0 & 0,275 & 0,725 & 0 & 0,3 & 0,7 & 0 & 0,325 & 0,675 & 0 & 0,35 & 0,65 & 0 & 0,375 & 0,625 & 0 \\
\hline 0,25 & 0,75 & 0 & 0,275 & 0,7025 & 0,0225 & 0,3 & 0,66 & 0,04 & 0,325 & 0,6225 & 0,0525 & 0,35 & 0,59 & 0,06 & 0,375 & 0,5625 & 0,0625 \\
\hline 0,25 & 0,75 & 0 & 0,275 & 0,7 & 0,025 & 0,3 & 0,65 & 0,05 & 0,325 & 0,6 & 0,075 & 0,35 & 0,55 & 0,1 & 0,375 & 0,5 & 0,125 \\
\hline 0,25 & 0,5 & 0,25 & 0,275 & 0,5225 & 0,2025 & 0,3 & 0,54 & 0,16 & 0,325 & 0,5525 & 0,1225 & 0,35 & 0,56 & 0,09 & 0,375 & 0,5625 & 0,0625 \\
\hline 0,25 & 0,5 & 0,25 & 0,275 & 0,5 & 0,225 & 0,3 & 0,5 & 0,2 & 0,325 & 0,5 & 0,175 & 0,35 & 0,5 & 0,15 & 0,375 & 0,5 & 0,125 \\
\hline 0,25 & 0,5 & 0,25 & 0,275 & 0,475 & 0,25 & 0,3 & 0,45 & 0,25 & 0,325 & 0,425 & 0,25 & 0,35 & 0,4 & 0,25 & 0,375 & 0,375 & 0,25 \\
\hline 0 & 1 & 0 & 0,05 & 0,95 & 0 & 0,1 & 0,9 & 0 & 0,15 & 0,85 & 0 & 0,2 & 0,8 & 0 & 0,25 & 0,75 & 0 \\
\hline 0 & 0,75 & 0,25 & 0,05 & 0,725 & 0,225 & 0,1 & 0,7 & 0,2 & 0,15 & 0,675 & 0,175 & 0.2 & 0,65 & 0,15 & 0,25 & 0,625 & 0,125 \\
\hline 0 & 0,75 & 0,25 & 0,05 & 0,7 & 0,25 & 0,1 & 0,65 & 0,25 & 0,15 & 0,6 & 0,25 & 0,2 & 0,55 & 0,25 & 0,25 & 0,50 & 0,25 \\
\hline
\end{tabular}

Or, d'après le modèle, seul le génotype femelle $\left(F_{h} e / F_{h} e\right)$ est capable, dans des croisements particuliers entre hermaphrodites et femelles, de donner des descendants en majorité hermaphrodites sans entraîner l'apparition de mâles (avec les génotypes possédant l'allèle $\mathrm{E}$ ).

$\mathrm{La}$ variété "Bicane" a donc pour génotype $\left(\mathrm{F}_{\mathrm{h}} \mathrm{e} / \mathrm{F}_{\mathrm{h}} \mathrm{e}\right)$.

\section{Exemple de la variété hermaphrodite "Counoise"}

La variété "Counoise" a la capacité d'induire une apparition importante de mâles, en plus d'hermaphrodites et de femelles, dans les descendants des croisements effectués entre elle et d'autres variétés hermaphrodites (TRUEL, comm. pers., 1977).

En outre, dans d'autres croisements du même type (TRUEL, comm. pers., 1977), les disjonctions sont exactement : $0,75(\emptyset)$ et $0,25(q)$. Or, d'après le modèle, seuls
2 génotypes sont capables d'induire une telle disjonction (autofécondations exclues) : $\left(\mathrm{F}_{\mathrm{h}} \mathrm{E} / \mathrm{F}_{\mathrm{h}} \mathrm{e}\right)$ et $\left(\mathrm{He} / \mathrm{F}_{\mathrm{m}} \mathrm{e}\right)$.

Pour trancher entre les 2 cas possibles, il faut examiner les disjonctions avec apparition de mâles pour ces mêmes types de croisements.

$\left(\mathrm{F}_{\mathrm{h}} \mathrm{E} / \mathrm{F}_{\mathrm{h}} \mathrm{e}\right)$ donne : soit $0,25\left(\sigma^{*}\right) ; 0,5(\delta)$ et $0,25(\%)$; ceci avec le génotype $\left(\mathrm{He} / \mathrm{F}_{\mathrm{m}} \mathrm{e}\right)$.

$\left(\mathrm{He} / \mathrm{F}_{\mathrm{m}} \mathrm{e}\right)$ donne :

soit $0,25(\delta) ; 0,5\left(\delta^{\star}\right)$ et $0,25(\%)$; évidemment avec $\left(\mathrm{F}_{\mathrm{h}} \mathrm{E} / \mathrm{F}_{\mathrm{h}} \mathrm{e}\right)$;

soit $0,25(\delta) ; 0,575\left(\delta^{\star}\right)$ et $0,175(\$) ;$ ceci avec $\left(\mathrm{F}_{\mathrm{h}} \mathrm{e} / \mathrm{HE}\right)$;

soit $0,25(\delta) ; 0,675(\phi)$ et $0,075(\%) ;$ ceci avec $\left(\mathrm{He} / \mathrm{F}_{\mathrm{h}} \mathrm{E}\right)$; 
soit $0,25(ठ) ; 0,75(\Varangle)$ et $0(\$)$; ceci avec (He/HE); soit $0,5\left(\delta^{*}\right) ; 0,5\left(\delta^{+}\right)$et $0(\%)$; ceci avec (HE/HE), $\left(F_{h} E / H E\right),\left(F_{h} E / F_{h} E\right)$.

Si l'on se reporte au tableau 1, le croisement des variétés "Counoise " et "Cabernet-Franc " ou "Aleatico " donne la disjonction suivante : $0,23(\delta), 0,52(\delta)$ et $0,25(\%)$, qui est très proche du $1^{\text {er }}$ type précédemment présenté ; mais ceci ne permet pas de choisir entre les 2 possibilités génotypiques pour la "Counoise».

Toutefois, il convient de noter le caractère assez exceptionnel du comportement de la variété «Counoise»par rapport à l'immense majorité des autres génotypes hermaphrodites (en particulier les variétés «Cabernet-Franc» et " Aleatico ») qui n'induisent jamais d'apparition de mâles dans les nombreux croisements que l'on a réalisés entre eux. Ceci est lié à la probabilité de réunion des allèles, $E$ et $F_{m}$ dans un même génotype. En outre, les déficits en femelles observables plus souvent dans ces mêmes types de croisements, sont dus à la réunion des ällèles $\mathrm{E}$ et $\mathrm{F}_{\mathrm{h}}$. Ceci revient à dire que très peu de variétés hermaphrodites possèdent l'allèle $F_{m}$ et que certaines d'entre elles possèdent l'allèle $E$. Les particularités exceptionnelles de la variété «Counoise » semblent donc plutôt liées à la possession de cet allèle $F_{m}$ qu'à celle de $\mathrm{E}$ et, parmi les génotypes hermaphrodites ayant ce dernier, une seule formule explique la disjonction sans mâles du type $0,75(\zeta)$ et $0,25(q)$ observée chez cette variété : $\left(\mathrm{He} / \mathrm{F}_{\mathrm{m}} \mathrm{e}\right)$. En conséquence, le génotype de la variété «Counoise» est $\left(\mathrm{He} / \mathrm{F}_{\mathrm{m}} \mathrm{e}\right)$, et celui des variétés "Cabernet-Franc" et "Aleatico " $\left(F_{h} E / F_{h} e\right)$.

Ces 2 exemples relatifs aux variétés "Bicane " et «Counoise » démontrent la possibilité de la détermination génotypique simple de quelques variétés. Dans la plupart des cas, le problème est plus compliqué et la solution fait appel à la fois à plusieurs séries de croisements « $F_{1} »$ avec la variété à identifier ainsi qu'à des « $F_{2}$ » entre celle-ci et les descendants « $F_{1}$ ». Ceci paraît réalisable pour les génotypes hermaphrodites et femelles, mais beaucoup plus difficile pour les génotypes mâles potentiellement très nombreux. En tout cas, les génotypes précédemment déterminés peuvent servir de révélateurs d'autres génotypes en limitant d'emblée le nombre de cas possibles à envisager.

\section{E. Intervention du dosage des allèles $E$ ou $F_{m}$}

Plusieurs observations supplémentaires sont de nature à affiner encore le modèle génétique précédemment proposé.

Tout d'abord, au sein d'un type floral mâle, hermaphrodite ou femelle, il existe des fluctuations morphologiques assez nettes (LeVAdOUX, 1946). Ceci n'est pas expliqué par le modèle puisque les allèles $\left(M, H, F_{m}, F_{h}, E\right)$ étant supposés posséder une efficacité totale, il n'existe qu'un phénotype mâle, un hermaphrodite et un femelle. Il faut donc faire intervenir un effet modificateur du milieu ou bien du génotype (multiallélisme accru aux mêmes loci ou gènes modificateurs supplémentaires). D'autres faits peuvent permettre de privilégier certaines hypothèses en la matière.

Il a été observé par ailleurs une conversion d'individus mâles en individus hermaphrodites soit sous l'effet de la vigueur de la plante stimulée par un milieu favorable, soit par application sur le pistil d'une cytokinine, la benzyl amino purine (NEGI \& OLMO, 1966). Ces 2 phénomènes peuvent d'ailleurs être reliés puisqu'une forte vigueur est en grande partie le résultat de l'activité des racines, au niveau de l'absorption de l'eau et des minéraux dans le sol, mais aussi à celui de la synthèse et de l'exportation de cytokinines dans la plante entière.

Ceci signifierait, soit que la stérilité femelle serait en fait un processus d'inhibition de la synthèse ou de l'action des cytokinines localisé dans le pistil, soit que l'adjonction massive de cytokinines sur le pistil créerait une voie artificielle de développement de cet organe en ne permettant qu'un fonctionnement très problématique au moment de la fécondation, ce qui est observé effectivement. Cette idée est en outre étayée par le fait que les cytokinines ont une action polyvalente dépassant le cadre de la stérilité. Il semblerait donc que l'hypothèse la plus vraisemblable serait celle de l'effet physiologique modificateur des cytokinines à un stade peu précisé du développement du sac embryonnaire, conséquence de la vigueur de la plante ou bien d'un traitement localisé, qui pourrait expliquer à la fois la «féminisation » des. mâles (NEGI \& OLMO, 1966) ainsi que les différents types morphologiques décrits par LEVADOUX (1946) ; ces derniers ne paraissent d'ailleurs pas se transmettre dans les descendances.

D'autres faits par contre semblent plutôt de nature génétique et sont en mesure d'affiner directement le modèle proposé. Le premier concerne certaines observations de TRUEL (comm. pers., 1977) consignées dans le tableau 1. Quelques types floraux, fonctionnellement hermaphrodites, ne sont en fait capables que d'une très rare nouaison: ce sont les « mâles - hermaphrodites ». Le phénomène de la coulure des fruits est-il une conséquence de l'efficacité de certains gènes proposés dans le modèle ou bien est-il le résultat de l'action d'autres gènes ? Cette question revêt un intéressant caractère d'applicabilité à la sélection de nouvelles variétés. Une autre observation va permettre de pousser plus loin l'analyse.

BRONNER (1981), en étudiant les descendants des croisements ou des autofécondations réalisés avec la variété très coularde «Muscat Ottonel», note une proportion importante de mâles. Pour l'autofécondation de cette variété, la disjonction est : $0,3(\zeta), 0,54(\delta)$ et $0,16(\$)$. Ce dernier résultat ne peut logiquement s'expliquer que si l'on suppose un effet de dosage de l'allèle (appelé ici $E^{\prime}$ ) chez la variété "Muscat Ottonel", cette particularité allélique conditionnant en outre son caractère "coulard » très accusé et assez exceptionnel parmi les variétés de vigne cultivées. Une telle hypothèse ne peut se généraliser à l'ensemble du genre Vitis puisqu'ici il s'agit du seul cas connu où des mâles apparaissent dans une descendance d'autofécondation.

Le génotype du «Muscat Ottonel» doit posséder le couple $\left(E^{\prime} / \mathrm{e}^{\prime}\right)$ pour donner lieu à des effets de dosage dans la descendance d'autofécondation, au moins un allèle $F_{m}$ pour expliquer l'apparition des mâles en liaison avec le couple $\left(E^{\prime}+E^{\prime}\right)$, et exclure l'allèle $M$. D'autre part, le génotype en question, qui est hétérozygote $\left(\mathrm{E}^{\prime} / \mathrm{e}^{\prime}\right)$ et qui a obligatoirement $F_{m}$, doit posséder $H$ ou $F_{h}$, sinon il serait femelle et non hermaphrodite. Quatre solutions sont donc possibles : $\left(\mathrm{HE}^{\prime} / \mathrm{F}_{\mathrm{m}} \mathrm{e}^{\prime}\right),\left(\mathrm{He}^{\prime} / \mathrm{F}_{\mathrm{m}} \mathrm{E}^{\prime}\right),\left(\mathrm{F}_{\mathrm{h}} \mathrm{E}^{\prime} / \mathrm{F}_{\mathrm{m}} \mathrm{e}^{\prime}\right)$ ou $\left(\mathrm{f}_{\mathrm{h}} \mathrm{e}^{\prime} / \mathrm{F}_{\mathrm{m}} \mathrm{E}^{\prime}\right)$. Toutefois, de tels modèles n'expliquent pas la disjonction observée par BRONNER (1981), par défaut de mâles, sauf si le taux de recombinaison entre les loci est voisin de 0 .

Il est plus logique de supposer par exemple un effet de dosage $(E+E)$ expliquant des recombinaisons mais portant sur un autre allèle que $F_{m}$, soit $F_{m}$. Le modèle suivant explique une disjonction d'autofécondation d'hermaphrodite du type : $0,25(\delta), 0,5(\delta), 0,25(q)$ : le génotype du "Muscat Ottonel », $\left(\mathrm{F}_{\mathrm{m}}^{\prime} \mathrm{E} / \mathrm{F}_{\mathrm{m}}^{\prime} \mathrm{e}\right)$, serait normalement mâle s'il s'agissait de l'allèle $F_{m}$, mais est hermaphrodite morphologiquement en manifestant une forte propension à la 
coulure avec $\mathrm{F}_{\mathrm{m}}$, ceci avec les relations suivantes :

$$
(E+E), F_{m}^{\prime}=M ;(E+e), F_{m}^{\prime}=H
$$

(coulard ou intermédiaire $« \mathrm{M}-\mathrm{H} »)$;

$$
(\mathrm{e}+\mathrm{e}), \mathrm{F}_{\mathrm{m}}^{\prime} \rightarrow \mathrm{F} \text {. }
$$

Il faut donc supposer, dans le genre Vitis, l'existence au même locus des allèles suivants : $M, H, F_{m}, F_{h}$ avec un effet épistatique de $E$ sur $F_{m}, F_{h}$ et, pour les cas exceptionnels de génotypes « coulards », la présence de l'allèle $F_{m}$ donnant un phénotype intermédiaire entre le mâle et l'hermaphrodite mais capable de donner le phénotype mâle en présence du couple $(E+E) . F_{m}^{\prime}$ serait donc à effet moins « féminisant $\gg$ que $\mathrm{F}_{\mathrm{m}}$.

Des observations ultérieures portant sur des cépages particuliers et mettant en évidence des disjonctions originales pourraient être explicables par une complexité accrue du nombre et des fonctions alléliques comme dans le cas précédent, sans remettre en cause les lois les plus générales. De même certaines déviations par rapport au modèle proposé pourraient exceptionnellement trouver une explication dans des biais liés à l'inévitable sélection pour la vigueur au sein des descendances ainsi que dans la présence de certaines chimères périclines.

Les conséquences de cette modélisation sont développées dans l'article suivant. Mais de toutes façons il conviendrait que les sélectionneurs de variétés de vigne apportent plus de précision encore au modèle génétique proposé.

Reçu le 2 août 1982 Accepté le 4 mars 1983.

\section{REMERCIEMENTS}

Nous tenons à remercier pour la communication de leurs observations: P. Truel (I.N.R.A., Domaine de Vassal, à Marseillan-Plage), J. P. Doazan, Mme Ottenwaelter, L. Bordenave (I.N.R.A., Station de Recherches, de Viticulture, Centre de Recherches de Bordeaux, à Pont-de-la-Mayc) et R. WAGNER (I.N.R.A., Station de Recherches viticoles à Villeneuve-lesMaguelonne).

\section{RÉFÉRENCES BIBLIOGRAPHIQUES}

Antcliff A. J., 1980. Inheritance of sex in Vitis. Ann. Amélior Plantes, 30, 113-122.

Breider H., Scheu H., 1937. Die Bestimmung und Vererbung des Geschlechts innerhalb der Gattung Vitis. Gartenbauwiss., 11, 627 674.

Bronner A., 1981. Observation de typcs sexuels mâles dans la descendance par autofécondation de la variété " Muscat Ottoncl " (Vitis vinifera L.). Vitis, 20, 211-217.

Doazan J. P., Cuellar V., 1970. Modification artificiclle de l'expression du sexe dans le genre Vitis. Ann. Amélior. Plantes, 20, 79.86.

Doazan J. P., Rives M., 1967. Sur la détermination génétique du scxe dans le genre Vitis. Ann. Amélior. Plantes, 17, 105-111.

Gorodea G. R., Neagu M. I., 1977. Contributii la studiul eredităth sexului la vita de ire. An. Inst. Vitic. Vinif., 8, 59-70.

Gorodea G. R., Neagu M. I., 1978. Aptitude à la combinaison du cépage «Bicanc». Génétique et Amćlioration de la Vignc. II' Symp. Intern. Amélior. Vigne, Bordeaux, 14-18 juin 1977. Ed. I.N.R.A. Paris, 97-104.

Levadoux L., 1946. Etude de la flcur ct de la scxualité chez la vigne. Ann. Ec. natl. Agric. de Montpellier, 27, 1-89.
Loomis N. H., 1948. A note on the inheritance of flower type in Muscadine grapes. Proc. am. Soc. Hort. Sci., 52, 276-278.

Loomis N. H., Williams C. F., 1954. Inheritance of flower types in Muscadine grapes. Proc. am. Soc. Hort. Sci., 64, 279-283.

Loomis N. H., Williams C. F., 1957. A new genetic flower type of the Muscadine grape. J. Hered., 48, 294-304.

Negi S. S., Olmo H. P., 1966. Scx conversion in a male Vitis vinifera $L$. by a kinin. Science, 152, 1624-1625.

Negi S. S., Olmo H. P., 1971. Conversion and detcrmination of sex in Vitis vinifera L. (sylvestris). Vitis, 9, 265-279.

Negrul A. M., 1930. Chromosomenzahl und Charakter der Reduktionsteilung bei den Artbastarden der Weinrebe (Vitis). Züchter, 2, 33-43.

Oberle G. O., 1938. A genetic study of variations in floral morphology and function in cultivated forms of Vitis. N.Y. Agric. Exp. Sta. Tech. Bull., 250, 1-63.

Tsekhmistrenko, Filinova, 1972. Sel'sko-Khoz Skok. Biol., 7, 356361.

Valleau W. D., 1916. Inheritance of sex in the grape. Am. Naturalist, 50, 554-561. 